\title{
Efeito da Utilização de Oligossacarídeo Manose e Acidificantes sobre o Desempenho de Coelhos em Crescimento
}

\author{
Claudio Scapinello', Haroldo Garcia de Faria ${ }^{2}$, Antônio Claudio Furlan ${ }^{1}$, Andrea Cristiane Michelan³
}

RESUMO - Foi conduzido um experimento com o objetivo de se determinar os efeitos da adição às rações de ácido fumárico, Oligossacarídeo manose $\left(\right.$ BIO-MOS ${ }^{\circledR}$ ), acidificante (ACID-ALL ${ }^{\circledR}$ ) e a combinação de oligossacarídeo com acidificantes sobre o desempenho de coelhos em crescimento. Foram utilizados 100 coelhos da raça Nova Zelândia Branco, 50 machos e 50 fêmeas, de 39 a 75 dias de idade, distribuídos em um delineamento experimental inteiramente casualizado com cinco tratamentos e 10 repetições e dois animais por unidade experimental. Considerando-se os períodos analisados 39-50 dias de idade e 39-75 dias, nenhuma das características de desempenho estudada foi afetada pela adição de oligossacarídeo, acidificantes ou a combinação de ambos.

Palavras-chave: acidificante, coelhos, desempenho, oligossacarídeo

\section{Effect of the Utilization of Oligosaccharide Mannose and Acidifiers on Growing Rabbits Performance}

\begin{abstract}
A trial was carried out to verify the effects of the addition of fumaric acid, oligosaccharide mannose (BIO-MOS ${ }^{\circledR}$ ), acidifiers (ACID-ALL ${ }^{\circledR}$ ) and oligosaccharide + acidifiers in diets growing rabbits on performance. One hundred NZW rabbits, 50 males and 50 females from 39 to 75 days old, distributed in a randomized experimental design with five treatments, 10 replications and two animals by experimental unit. In the period from 39-50 and 39-75 days of experiment none of these performance characteristics was affected by inclusion of oligosaccharide mannose, acidifiers and oligosaccharide +acidifiers did not influence the performance of growing rabbits.
\end{abstract}

Key Words: acidifiers, oligosaccharide, performance, rabbits

\section{Introdução}

Para que a cunicultura seja uma atividade rentável é necessário reduzir o custo da alimentação e diminuir a mortalidade, especialmente até os 50 dias de idade (GIPPERT et al., 1996). A ocorrência de enterites, principalmente logo após a desmama, é a maior causa de perdas econômicas numa exploração comercial de coelhos. Segundo PEETERS et al. (1984), as causas da elevada mortalidade incluem viroses, bactérioses, coccídeoses, assim como fatores dietéticos, em especial o nível e a composição da fibra e a sua relação com carboidratos solúveis.

A microbiota do trato digestivo possui uma ação significativa no estado sanitário dos animais e nos parâmetros zootécnicos dos mesmos. Nesse sentido, tem surgido a idéia da biorregulação da microbiota que permitiria otimizar os resultados e minimizar as perdas de animais.

A indústria alimentícia utiliza dois tipos de substância que atuam de forma a modificar a flora: os probióticos e os antibióticos.

Neste sentido, os oligossacarídeos aparecem como uma nova via que oferece perspectivas de estudos. Suplementação com oligossacarídeos às dietas de coelhos provoca aumentos nos níveis cecais de ácidos graxos voláteis e, em coelhos desmamados, diminui a amônia cecal. Estas mudanças podem ajudar na prevenção de colibaciloses (PEETERS et al., 1992).

Oligossacarídeos são cadeias longas de açúcares simples como manose ou frutose. Os oligossacarídeos de frutose e oligossacarídeos de manose são elementos aditivos de rações para animais não ruminantes. Porém, estes elementos aditivos operam por mecanismos diferentes.

Os oligossacarídeos de frutose são produtos da indústria que, adicionado às rações fornecem carboidratos fermentáveis para as bactérias benéficas nativas que habitam o trato gastrointestinal. De acordo com esta teoria, o efeito benéfico dos microorganismos providos com quantias adequadas

\footnotetext{
1 Professores do Departamento de Zootecnia/UEM - Av. Colombo 5790, Maringá - PR, Cep 87020-900. E.mail: cscapinello@uem.br 2 Zootecnista do Biotério Central - UEM.

3 Estudante do curso de Pós-Graduação em Zootecnia - UEM.
} 
de carboidratos fermentáveis, seria que estes minimizariam as populações de bactérias patogênicas, como a Escherichia coli e Salmonella, por exclusão competitiva.

O oligossacarídeo de manose opera por um mecanismo mais complexo. Este é derivado da parede celular interna de culturas de leveduras e o primeiro modo de atuação é ligando-se a certas bactérias patogênicas na área gastrointestinal; essas bactérias ligadas aos oligossacarídeos não podem aderir à infecção iniciada no intestino. Porém, algumas bactérias não possuem em suas membranas celulares sítios de ligação para fixação dos oligossacarídeos, como por exemplo a (clostridia) bactéria que causa a enterite necrótica no intestino; no entanto, a concentração desta bactéria é reduzida quando os oligossacarídeos manose são administrados. Neste caso, o segundo mecanismo de atuação dos oligossacarídeos manose seria a modulação ou preparação do sistema imune para uma infecção.

Acidificantes são ácidos orgânicos que têm efeitos benéficos quando somado à dieta de animais desmamados (EDMONDS et al., 1985). A adição de ácidos orgânicos na dieta animal melhora a eficiência alimentar e a velocidade de crescimento (PATTEN e WALDROUP, 1988). Ácidos orgânicos têm demonstrado ótimos resultados em várias espécies animais, principalmente em animais jovens e adultos em estado de estresse (HOLLISTER et al.,1989).

Em animais recém desmamados, o sistema digestivo se apresenta relativamente imaturo. Como conseqüência, estes não conseguem digerir, no intestino delgado, os carboidratos e proteínas contidos nos grãos de cereais e sementes oleaginosas tão eficientemente como os carboidratos do leite (lactose) e as proteínas do leite (caseína, lactoalbuminas). Isto se deve, em parte, às secreções insuficientes de enzimas e à produção de ácido clorídrico pelo estômago (CROMWELL, 1989). Segundo SEERLEY (1994), a administração de agentes acidificantes apresenta efeitos benéficos para a saúde do trato digestivo.

O desenvolvimento de resistência bacteriana e a exigência por parte dos importadores que desejam produtos livres de resíduos de aditivos permitiram que o uso de oligossacarídeos, juntamente com os acidificantes, merecem atenção por parte de pesquisadores como uma possível alternativa ao uso dos tradicionais promotores de crescimento. Dessa forma, tornam-se necessário mais estudos no sentido de buscar esclarecimentos adicionais sobre a viabilidade do uso destas substâncias na nutrição animal.
O objetivo do presente experimento foi avaliar o efeito da adição de oligossacarídeo manose e acidificantes às dietas sobre o desempenho de coelhos em crescimento.

\section{Material e Métodos}

Foram utilizados 100 coelhos da raça Nova Zelândia Branco, 50 machos e 50 fêmeas, 39-75 dias de idade, alojados em gaiolas de arame galvanizado providas de bebedouros automático e comedouro semi-automático de chapa galvanizada, localizados em galpão de alvenaria, com cobertura de telha francesa, pé-direito de 3,0 metros, piso em alvenaria, paredes laterais de $50 \mathrm{~cm}$ em alvenaria e o restante em tela e cortina de plástico para controle de ventos.

Os animais foram distribuídos em um delineamento experimental inteiramente casualizado com cinco tratamentos e 10 repetições com dois animais por unidade experimental. As dietas foram formuladas de acordo com as recomendações do AEC (1987) para coelhos em crescimento. Os tratamentos eram compostos de uma dieta referência e 4 dietas com adição de oligossacarídeo manose, acidificantes e combinação de ambos. Os acidificantes usados foram: ácido fumárico, ACID ALL ${ }^{\circledR}$ cuja composição básica do produtoé formada por: ácido sórbico, ácido fosfórico, ácido lático, ácido fumárico, ácido propiônico, calcário e silicato de alumínio. O oligossacarídeo manose usado foi o BIO-MOS ${ }^{\circledR}$ derivado da cultura de Saccharomyces cerevisea.

O premix vitamínico mineral utilizado era desprovido de promotor de crescimento, coccidiostático ou antibiótico, com o objetivo de evitar interferências destes produtos nos processos de digestão e/ou desempenho dos animais. As composições das dietas experimentais são mostradas na Tabela 1 .

Após misturadas, as rações foram peletizadas a seco e o fornecimento das rações e água foi à vontade.

Os coelhos foram pesados no início do experimento com 39 dias de idade, aos 50 dias e no final do experimento aos 75 dias de idade. As rações fornecidas e as sobras também foram pesadas a cada pesagem dos animais.

O abate dos animais, no final do experimento, foi realizado conforme descrição feita por SCAPINELLO (1993).

As características avaliadas referentes à carcaça foram obtidas com a carcaça quente, sem a cabeça.

O modelo estatístico utilizado para análise das características de desempenho de carcaça foi: 
1274 Rev. bras. zootec.

Tabela 1 - Composição percentual das dietas experimentais

Table 1 - Percentage composition of the experimental diets

\begin{tabular}{|c|c|c|c|c|c|c|}
\hline $\begin{array}{l}\text { Ingredientes } \\
\text { Ingredients }\end{array}$ & $\begin{array}{c}\text { Unidade } \\
\text { Unit }\end{array}$ & $\begin{array}{l}\text { Teste } \\
\text { Control }\end{array}$ & $\begin{array}{l}\text { Ácido fumárico } \\
\text { Fumaric acid }\end{array}$ & ACID ALL $^{\circledR}$ & BIO-MOS $^{\circledR}$ & $\begin{array}{l}\text { BIO-MOS }^{\circledR}+ \\
\text { ACID-ALL }^{\circledR} \\
\end{array}$ \\
\hline Milho & $\mathrm{kg}$ & 28,87 & 28,87 & 28,87 & 28,87 & 28,87 \\
\hline $\begin{array}{l}\text { Farelo de soja } \\
\text { Soybean meal }\end{array}$ & $\mathrm{kg}$ & 13,00 & 13,00 & 13,00 & 13,00 & 13,00 \\
\hline $\begin{array}{l}\text { Farelo de trigo } \\
\text { Wheat meal }\end{array}$ & $\mathrm{kg}$ & 19,00 & 19,00 & 19,00 & 19,00 & 19,00 \\
\hline $\begin{array}{l}\text { Feno de alfafa } \\
\text { Alfafa hay }\end{array}$ & $\mathrm{kg}$ & 25,00 & 25,00 & 25,00 & 25,00 & 25,00 \\
\hline $\begin{array}{l}\text { Feno de aveia } \\
\text { Oats hay }\end{array}$ & $\mathrm{kg}$ & 10,00 & 10,00 & 10,00 & 10,00 & 10,00 \\
\hline $\begin{array}{l}\text { Sal comum } \\
\text { Salt }\end{array}$ & $\mathrm{kg}$ & 0,40 & 0,40 & 0,40 & 0,40 & 0,40 \\
\hline $\begin{array}{l}\text { Fosfato bicálcico } \\
\text { Dicalcium phosphat }\end{array}$ & $\mathrm{kg}$ & 0,50 & 0,50 & 0,50 & 0,50 & 0,50 \\
\hline $\begin{array}{l}\text { Calcário } \\
\text { Limestone }\end{array}$ & $\mathrm{kg}$ & 1,00 & 1,00 & 1,00 & 1,00 & 1,00 \\
\hline $\begin{array}{l}\text { DL-Metionina } \\
\text { DL-Methionine }\end{array}$ & $\mathrm{kg}$ & 0,10 & 0,10 & 0,10 & 0,10 & 0,10 \\
\hline $\begin{array}{l}\text { Mist. Vit+Min }{ }^{1} \\
\text { Premix vit+min. }{ }^{1}\end{array}$ & $\mathrm{~kg}$ & 0,50 & 0,50 & 0,50 & 0,50 & 0,50 \\
\hline $\begin{array}{l}\text { Inerte } \\
\text { Inert }\end{array}$ & $\mathrm{kg}$ & 1,63 & 0,13 & 1,48 & 1,48 & 1,43 \\
\hline $\begin{array}{l}\text { Ácido fumárico } \\
\text { Fumaric acid }\end{array}$ & $\mathrm{kg}$ & - & 1,50 & - & - & - \\
\hline $\begin{array}{l}\text { ACIDALL }{ }^{\circledR} \\
\text { BIO-MOS }\end{array}$ & $\begin{array}{l}\mathrm{kg} \\
\mathrm{kg}\end{array}$ & $\begin{array}{l}- \\
-\end{array}$ & $\begin{array}{l}- \\
-\end{array}$ & 0,150 & $\overline{0,150}$ & $\begin{array}{l}- \\
-\end{array}$ \\
\hline $\begin{array}{l}\text { ACIDALL }^{\circledR}+ \\
\text { BIO-MOS }^{\circledR} \\
\text { Composição calcul } \\
\text { Calculated compositi }\end{array}$ & $\begin{array}{l}\mathrm{kg} \\
\mathrm{da}\end{array}$ & - & - & - & - & 0,200 \\
\hline $\begin{array}{l}\text { Matéria seca } \\
\text { Dry matter }\end{array}$ & $\%$ & 89,33 & 89,33 & 89,33 & 89,33 & 89,33 \\
\hline $\begin{array}{l}\text { Proteína bruta } \\
\text { Crude protein }\end{array}$ & $\%$ & 17,00 & 17,00 & 17,00 & 17,00 & 17,00 \\
\hline $\begin{array}{l}\text { FDN } \\
N D F\end{array}$ & $\%$ & 27,40 & 27,40 & 27,40 & 27,40 & 27,40 \\
\hline $\begin{array}{l}\text { FDA } \\
A D F\end{array}$ & $\%$ & 16,00 & 16,00 & 16,00 & 16,00 & 16,00 \\
\hline $\begin{array}{l}\text { Cálcio } \\
\text { Calcium }\end{array}$ & $\%$ & 0,5 & 0,5 & 0,5 & 0,5 & 0,5 \\
\hline $\begin{array}{l}\text { Fósforo } \\
\text { Phosphorus }\end{array}$ & $\%$ & 1,00 & 1,00 & 1,00 & 1,00 & 1,00 \\
\hline $\begin{array}{l}\text { Energia digestível } \\
\text { Digestible energy }\end{array}$ & $\mathrm{kcal} / \mathrm{kg}$ & 2570 & 2570 & 2570 & 2570 & 2570 \\
\hline
\end{tabular}

$$
\mathrm{Y}_{i j}=\mathrm{m}+\mathrm{D}_{i}+\mathrm{e}_{i j}
$$

em que: $\mathrm{Y}_{i j}=$ valor observado das variáveis estudadas dos animais $j$, que receberam dieta $i$; $\mu=$ constante geral $; D_{i}=$ efeito da dieta experimental $i$, sendo $i=1,2,3,4$ e $5(i 1=$ dieta referência, $i 2=$ dieta com $1,5 \%$ de ácido fumárico, $i 3$ dieta contento $0,15 \%$ de ACID ALL ${ }^{\circledR}$, i4 dieta contendo $0,15 \%$ de
BIO-MOS ${ }^{\circledR}$ e $i 5$ dieta contendo a combinação de $0,10 \%$ ACID ALL ${ }^{\circledR}$ e $0,10 \%$ BIO-MOS $\left.^{\circledR}\right) ; \mathrm{e}_{i j}=$ erro aleatório associado a cada observação.

Para análise estatística utilizou-se o SAEG, desenvolvido pela UNIVERSIDADE FEDERAL DE VIÇOSA (1997) e o teste de Tukey para comparação das médias. 


\section{Resultados e Discussão}

As médias de peso vivo aos 50 e 75 dias de idade, ganho de peso diário, consumo de ração diário e a conversão alimentar, no período de 39 a 50 e de 39 a 75 dias de idade, peso e rendimento de carcaça sem cabeça de acordo com os diferentes tratamentos, são mostradas nas Tabelas 2 e 3. Observou-se que, na presente condição experimental, a utilização de acidificantes e oligossacarídeos não promoveu melhora $(\mathrm{P}>0,05)$ sobre nenhuma das características avaliadas, tanto no período de 39 a 50 como no período total do experimento, de 39 a 75 dias.

Os resultados obtidos com acidificantes diferem daqueles encontrados por HOLLISTER et al. (1989), que, em estudo com coelhos desmamados, verificaram efeitos favoráveis sobre a conversão alimentar e diminuição na incidência de enterites. Também HOLLISTER et al. (1990), em um total de seis experimentos, estudando os efeitos de acidificantes (Acid-Park 4-Way®) em coelhos desmamados, verificaram redução de enterites e melhora na conversão alimentar.

CASTROVILLI (1991), trabalhando com coelhos mestiços de peso entre 900 e $1000 \mathrm{~g}$, com misturas de ácidos orgânicos e inorgânicos (ácido fosfórico, ácido acético, ácido lático, ácido tartárico e málico), concluiu que a adição de $0,3 \%$ destes ácidos à ração provocou melhoras no ganho de peso, na conversão alimentar e nos processos digestivos.

Também FALKOWSLKI et al. (1995) constataram melhora na conversão alimentar de suínos em crescimento com adição de 1,5 ou $2 \%$ de ácido fumárico na dieta. SCAPINELLO et al. (1999), trabalhando com coelhos de 40-80 dias, também verificaram melhora na conversão alimentar com adição de $2 \%$ de ácido fumárico às rações.

FARIA et al. (1999), trabalhando com coelhos alimentados com diferentes níveis de levedura de recuperação (Saccharomyces $s p$ ) em rações contendo ou não $2 \%$ de ácido fumárico, verificaram melhora na conversão alimentar, com a adição de $2 \%$ ácido fumárico às rações.

Em relação ao efeito do oligossacarídeo manose, não foi encontrado efeito $(\mathrm{P}>0,05)$ sobre as características estudadas. Estes resultados diferem dos encontrados por LEBAS (1993) e ROCA (1994), que, trabalhando com oligossacarídeos para coelhos, verificaram melhora na conversão alimentar.

MENDEZ et al. (1993), trabalhando com oligossacarídeo, constataram melhora no desempenho e na conversão alimentar de coelhos. Também AGUILAR (1996) e LEBAS (1996) observaram melhora no desempenho, quando foram adicionados oligossacarídeos às dietas de coelhos.

Contudo, é bom ressaltar que todos os autores citados realizaram seus trabalhos com oligossacarídeo frutose.

A falta de resposta positiva ao acidificante pode ser explicada por alguns fatores, entre os quais citam-se o tempo pós-desmama e o tipo e a dosagem do acidificante. Em relação, ao tempo pós-desmama, pode-se inferir que a resposta dos animais ocorre por um período pequeno após a desmama. GIESTING et al. (1991) afirmam que a resposta à acidificação é mais evidente nos primeiros dias após a desmama, declinando com a idade.

Os fatores relacionados com a dosagem do acidificante estão relacionados com sua constante de

Tabela 2 - Peso vivo aos 50 dias de idade (PV50), ganho de peso diário (GPD), consumo de ração diário (CRD) e conversão alimentar (CA) de coelhos de 39 a 50 dias de idade, de acordo com os diferentes tratamentos contendo acidificantes e oligosacarídeo nas rações

Table 2 - Means of live weight (LW), daily weight gain (DWG), daily ration consuption (DFI) and feed conversion (FC) of rabbits from 3950 days of age, according to different treatments of acidifiers and oligosaccharide in the diets

\begin{tabular}{lcccccc}
\hline $\begin{array}{l}\text { Tratamentos } \\
\text { Treatments }\end{array}$ & $\begin{array}{c}\text { Teste } \\
\text { Control }\end{array}$ & $\begin{array}{c}\text { Ac. fumárico } \\
\text { Fumaric ac. }\end{array}$ & ACID ALL $^{\circledR}$ & BIO-MOS $^{\circledR}$ & $\begin{array}{c}\text { BIO-MOS }^{\circledR}+ \\
\text { ACID ALL }^{\circledR}\end{array}$ & CV\% $^{{ }^{\circledR}}$ \\
\hline PV 50 $(\mathrm{g})$ & $1373^{\mathrm{A}}$ & $1442 \mathrm{~A}$ & $1419^{\mathrm{A}}$ & $1392^{\mathrm{A}}$ & $1407^{\mathrm{A}}$ & 5,47 \\
$L W$ & $38^{\mathrm{A}}$ & $44^{\mathrm{A}}$ & $42^{\mathrm{A}}$ & $39^{\mathrm{A}}$ & $41^{\mathrm{A}}$ & 17,12 \\
GPD $(\mathrm{g})$ & $92 \mathrm{~A}$ & $97^{\mathrm{A}}$ & $96^{\mathrm{A}}$ & $89^{\mathrm{A}}$ & $94^{\mathrm{A}}$ & 10,31 \\
CRD $(\mathrm{g})$ & $2,53^{\mathrm{A}}$ & $2,20^{\mathrm{A}}$ & $2,31^{\mathrm{A}}$ & $2,41^{\mathrm{A}}$ & $2,31^{\mathrm{A}}$ & 18,15 \\
$D F I$ & & & & & &
\end{tabular}

Médias com letras iguais no sentido de linha não diferem $(P>0,05)$ pelo teste de Tukey.

Means, in a row, followed by the same letters do not differ $(P>.05)$ by Tukey test. 
1276 Rev. bras. zootec.

Tabela 3 - Peso vivo aos 75 dias de idade (PV75), ganho de peso diário (GPD), consumo de ração diário (CRD), conversão alimentar (CA), peso de carcaça (PC) e rendimento de carcaça (RC) de coelhos de 39 a 75 dias de idade, de acordo com os diferentes tratamentos contendo acidificantes e oligosacarideo nas rações

Table 3 - Means of live weight (LW), daily weight gain (DWG), daily feed intake (DFI), feed: gain ratio (FC), carcass weight (CW) and carcass yield $(C Y)$ of rabbits from 39-75 days of age, according to different treatments of acidifiers and oligosaccharide in the diets

\begin{tabular}{|c|c|c|c|c|c|c|}
\hline $\begin{array}{l}\text { Tratamentos } \\
\text { Treatments }\end{array}$ & $\begin{array}{l}\text { Teste } \\
\text { Control }\end{array}$ & $\begin{array}{l}\text { Ac. fumárico } \\
\text { Fumaric ac. }\end{array}$ & ACID ALL $^{\circledR}$ & BIO-MOS $^{\circledR}$ & $\begin{array}{l}\text { BIO-MOS }^{\circledR}{ }_{+} \\
\text {ACID ALL }^{\circledR}\end{array}$ & CV\% \\
\hline $\begin{array}{l}\mathrm{PV} 75(\mathrm{~g}) \\
L W\end{array}$ & $1953^{\mathrm{A}}$ & $2065^{\mathrm{A}}$ & $1974^{\mathrm{A}}$ & $1957^{\mathrm{A}}$ & $2055 \mathrm{~A}$ & 8,00 \\
\hline $\begin{array}{l}\mathrm{GPD}(\mathrm{g}) \\
D W G\end{array}$ & $28^{\mathrm{A}}$ & $31^{\mathrm{A}}$ & $28^{\mathrm{A}}$ & $28^{\mathrm{A}}$ & $30^{\mathrm{A}}$ & 15,35 \\
\hline $\begin{array}{l}\mathrm{CRD}(\mathrm{g}) \\
D F I\end{array}$ & $92^{\mathrm{A}}$ & $98^{\mathrm{A}}$ & $91^{\mathrm{A}}$ & $88^{\mathrm{A}}$ & $96^{\mathrm{A}}$ & 13,07 \\
\hline $\begin{array}{l}\mathrm{CA} \\
F C\end{array}$ & $3,34^{\mathrm{A}}$ & $3,21^{\mathrm{A}}$ & $3,24^{\mathrm{A}}$ & $3,20^{\mathrm{A}}$ & $3,16^{\mathrm{A}}$ & 7,06 \\
\hline $\begin{array}{l}\mathrm{PC}(\mathrm{g}) \\
C W\end{array}$ & $1035^{\mathrm{A}}$ & $1115^{\mathrm{A}}$ & $1047^{\mathrm{A}}$ & $1037^{\mathrm{A}}$ & $1109^{\mathrm{A}}$ & 11,05 \\
\hline $\begin{array}{l}\mathrm{RC}(\%) \\
C Y\end{array}$ & $53^{\mathrm{A}}$ & $54^{\mathrm{A}}$ & $53^{\mathrm{A}}$ & $53^{\mathrm{A}}$ & $54^{\mathrm{A}}$ & 4,62 \\
\hline
\end{tabular}

Médias com letras iguais no sentido de linha não diferem $(P>0,05)$ pelo teste de Tukey.

Means, in a row, followed by the same letters do not differ $(P>.05)$ by Tukey test.

dissociação que identifica sua capacidade acidificante. GARDENER (1972) afirma que a constante de dissociação e a solubilidade em água dos diferentes ácidos orgânicos podem ser fatores que influenciam as respostas à acidificação.

Vários estudos foram realizados no sentido de se determinar o nível ótimo de dosagem de acidificantes em algumas espécies animais (FALKOWSKI e AHENE, 1984; PATTEN e WALDROUP, 1988; SCAPINELLO et al, 1998) porém, a resposta depende do tipo da dieta e da idade do animal, sendo que altas doses podem levar a uma resposta negativa no desempenho.

Em relação à falta de resposta ao oligossacarídeo manose, possivelmente as boas condições de higiene do ambiente bem como manejo e estado sanitário dos animais não tenham permitido resposta adicional no desempenho dos coelhos uma vez que o oligossacarídeo manose atua basicamente por exclusão de microorganismos prejudiciais, assegurando melhor utilização dos alimentos.

\section{Conclusões}

Nas condições em que o experimento foi realizado, a adição de acido fumárico, oligossacarídeo manose, acidificante e sua combinação com oligossacarídeo manose às rações não influenciaram o desempenho de coelhos em crescimento, porém mais estudos devem ser realizados no sentido de se verificar a viabilidade de uso destes produtos.

\section{Referências Bibliográficas}

AEC. 1987. Recomendações para nutrição animal.5.ed, RHÔNEPOULENC. 86p.

AGUILAR, J.C., ROCA, T., SANZ, E. Fructo-oligosaccharides in rabbit diets. Study of efficiency in suckling and fattening periods. In: WORLD RABBIT CONGRESS, 6, 1996, Tolouse, Proceedings...Tolouse, 1996. p.73-77.

CASTROVILLI, C. 1991.Acidificazione del mangine per conigli all'engrasso. Riv. di Conegl., 28(8):31-34.

CROMWELL, G.L. 1989. Nuevos aditivos alimenticios. Ind. Porcina, 9(6):25-36.

EDMONDS, S.M., IZQUIERDO, A.O., BAKER, H.D. 1985. Feeds additives with newly weaned pigs: efficacy of supplemental copper, antibiotics and organic acids. J. Anim. Sci., 60(2):462-469.

FALKOWSKI, J., AHENE, X.F. 1984. Fumaric and citric acid as feed additives in starter pig nutrition. J. Anim. Sci., 58(4):935-938

FALKOWSKI, J., MILEWSKA, W., KOZERA, FALKOWSKA, A. et al. 1995. Results of rearing and some blood indices of piglets fed on feed concentrates containing fumaric acid or probiotics acid sacc. Acta Acad. Agricult. Tech. Olstenensis, Zootechnica, 20 (43):33-41.

FARIA, H.G., SCAPINELLO, C. FURLAN, A.C. et al. 1999 Desempenho de coelhos em crescimento, alimentados com diferentes níveis de levedura de recuperação (Saccharomyces $s p$ ), seca por spray-dry, em rações contendo ou não ácido fumárico. Acta Scientiarum, 21(3):693-698.

GARDENER, W.H. 1972. Acidulants in food processing. In: FURIA, T.E. CLEVELAND, O.H. (Eds.) CRC Handbook of food additives.

GIESTING, D.W., ROSS, M.A., EASTER, R.A.1991. Evaluation of the effect of fumaric acid and sodium bicarbonate addition on performance of starter pigs fed diets differents types. J. Anim. Sci., 69:2489-2496.

GIPPERT,T., BERSENYI, A., SZABÓ, L. et al. 1996. Development of novel feed concentrates supplemented with 
SCAPINELLO et al.

salinomycin and lacto-sacc for growing rabbit nutrition in small scale farms. In: WORLD RABBIT CONGRESS, 6, 1996, Tolouse. Proceedings...Tolouse, 1996. v.1, p.187-190.

HOLLISTER, A.G., CHEEKE. P.R., ROBINSON, K.L. et al. 1989. Effects of water-administered probiotics and acidifiers on growth, feed conversion and enteritis mortality of weanling rabbits. J. Appl. Rabbit Res., 12:143-147.

HOLLISTER, A.G., CHEEKE. P.R., ROBINSON, K.L. et al. 1990. Effects of dietary probiotics and acidifiers on performance of weanling rabbits. J. Appl. Rabbit Res., 13:6-9.

LEBAS, F. 1993. Incidence du Profeed sur l'efficaté alimenatire chez de lapin en croissance. Cuniculture, 20(112):169-173

LEBAS, F. 1996. Effects of fructo-oligosaccharides origin on rabbits growth performance in 2 seasons In: WORLD RABBIT CONGRESS, 6, Tolouse. Proceedings...Tolouse, 1996. v.1., p.211-215

MENDEZ, J., MATEOS, G.G., TABOADA, S., GROBAS, S. Utilizacion de fructooligosaccharides (Profeed) em piensos de engorde de conejos. In: SIMPÓSIO DE CUNICULTURA, 18, 1993. p.69-70.

PATTEN, J.D., WALDROUP, P.W. 1988. Use of organic acids in broiler diets. Pout. Sci., 67(6):1178-1182.

PEETERS, J.E., POHL, P., CHARLIER, G. et al. 1984. Infections agents associated with diarrhea in commercial rabbits: a field study. In: WORLD RABBIT CONGRESS, 3, Roma, Proceedings... Roma, 1984. v.2, p.265-273.

PEETERS, J.E., MAERTENS, L., GEEROMS, R. 1992. Influence of galactoolisaccharides on zootechnical performance, cecal biochemistry and experimental colibacilosis in weanling rabbits. J. Appl. Rabbit Res., 15:1129-1136.
ROCA, T. 1994. Efecto mejorante de l'adicion del fructooligosacarideo Profeed en el pienso de engorde de conejos. Boletín de Cunicultura, 17(5):41-45

SEERLEY, R.W. 1994. A importância da acidificação na nutrição de monogástrico. In: Proceedings of altech's annual syposium. Nicholasville, Altech Technical Publications. p.79-88.

SCAPINELLO, C. Níveis de proteína bruta e de energia digestivel e exigências de lisina e de metionina + cistina para coelhos da raça Nova Zelândia Branco em crescimento. Viçosa, MG: UFV, 1993. Tese (Doutorado em Produção Animal) - Universidade Federal de Viçosa, 1993.

SCAPINELLO, C., FARIA, H.G., FURLAN, A.C. et al. 1998. Influencia de diferentes níveis de ácido fumárico ou ácido acético sobre o desempenho de coelhos em crescimento. Rev. Bras. Zootec., 27(5):935-940.

SCAPINELLO, C., FARIA, H.G., FURLAN, A.C. 1999. Influencia de diferentes níveis de ácido fumárico sobre o desempenho de coelhos em crescimento. Rev. Bras. Zootec., 28(4):785-790.

UNIVERSIDADE FEDERAL DE VIÇOSA - UFV. 1997. SAEG. Sistema de análises estatísticas e genéticas. Versão 7.1, Viçosa, MG, 150p. (Manual do usuário).

Recebido em: 01/12/00

Aceito em: 27/04/01 